\title{
Distributed localization using acoustic Doppler
}

\author{
David Lindgren, Gustaf Hendeby and Fredrik Gustafsson
}

\section{Linköping University Post Print}

\section{Tweet}

N.B.: When citing this work, cite the original article.

Original Publication:

David Lindgren, Gustaf Hendeby and Fredrik Gustafsson, Distributed localization using acoustic Doppler, 2015, Signal Processing, (107), 43-53.

http://dx.doi.org/10.1016/j.sigpro.2014.06.031

Copyright: Elsevier

http://www.elsevier.com/

Postprint available at: Linköping University Electronic Press

http://urn.kb.se/resolve?urn=urn:nbn:se:liu:diva-113503 


\title{
Distributed Localization using Acoustic Doppler
}

\author{
David Lindgren $^{\mathrm{a}, \mathrm{b}}$, Gustaf Hendeby ${ }^{\mathrm{a}, \mathrm{b}}$, Fredrik Gustafsson ${ }^{\mathrm{a}}$ \\ ${ }^{a}$ Dept. Electrical Engineering, Linköping University, SE-581 83 Linköping, Sweden. \\ ${ }^{b}$ Dept. of Sensor \& EW Systems, Swedish Defence Research Agency (FOI), SE-581 11, Linköping, Sweden
}

\begin{abstract}
It is well-known that the motion of an acoustic source can be estimated from Doppler shift observations. It is however not obvious how to design a sensor network to efficiently deliver the localization service. In this work a rather simplistic motion model is proposed that is aimed at sensor networks with realistic numbers of sensor nodes. It is also described how to efficiently solve the associated least squares optimization problem by Gauss-Newton variable projection techniques, and how to initiate the numerical search from simple features extracted from the observed frequency series. The methods are evaluated by Monte Carlo simulations and demonstrated on real data by localizing an all-terrain vehicle. It is concluded that the processing components included are fairly mature for practical implementations in sensor networks.
\end{abstract}

Keywords: Acoustic tracking, Sensor network, Acoustic Doppler, Localization, Variable projection

\section{Introduction}

The motion of a passing acoustic source can be estimated from the observed Doppler shift. We consider an acoustic sensor network with two or more synchronized nodes with known positions. It is assumed that the Doppler frequency of a passing acoustic source can be detected and measured by each sensor. It is further assumed that the source motion obeys a parameterized model. We address the problem of identifying the model parameters, from which the source trajectory is deduced.

The problem of localizing moving acoustic sources based on Doppler shift observations has received fairly much attention in the literature, especially during a period around 1990. Still, there are aspects within the area that are not entirely covered, and today it would be particularly interesting to revive the interest for the acoustic Doppler phenomenon with the background of both the last decade of advances in wireless sensor network technologies and new opportunities to solve the associated numerical problems with state-of-the-art algorithms.

A number of authors have considered the case with a single sensor by which the source passage distance and assumed constant speed are estimated [1-4]. It appears, however, that the estimation problem is inherently nonlinear

\footnotetext{
${ }^{*}$ Corresponding author

Email addresses: david. Iindgren@foi.se (David Lindgren), hendeby@isy. liu. se (Gustaf Hendeby), fredrik.gustafsson@liu.se (Fredrik Gustafsson)
} 
in the parameters, and a rather wide range of different numerical approaches are proposed to pursue the, in various senses, optimal estimate. Most commonly, the parameters are given by minimizing some variation of a least squares criterion.

A complication in the acoustic case is the retardation; the effect that, when the sound reaches the sensors, the source has already moved to a new position, see [5]. To avoid any misunderstanding regarding our terminology, note that the retardation effect is unrelated to any source velocity retardation. It is the phenomena that follow from retarded signals; signals that are delayed due to finite propagation speed. In the radar case, this effect can usually be disregarded since the propagation speed of the electromagnetic waves is many orders of magnitudes larger than the speed of most sources of practical interest (the "stop-and-go approximation"), see [6, pp. 97]. Disregarding the retardation in the acoustic case may, depending on geometry and source speed, however result in significant estimation bias.

The approximate retardation-free model can be parameterized with only two nonlinear parameters (instead of three or four), for instance, an $x$ and a $y$ coordinate on the source trajectory. This low parameter dimension facilitates an exhaustive grid search for the (least squares) minimum, which avoids otherwise problematic local minima. This is elegantly demonstrated by [2]. By also using the frequency time derivative as a measurement, the grid search can be conducted in only one dimension, see [7]. Actually, by disregarding the retardation, an exact solution based on a deterministic model and three frequency measurements can be found by calculating the roots of a third order polynomial, see [1].

Another estimation technique is to match an observed frequency series with a library of precomputed Doppler shift profiles. This way, a complex retardation model may be used as a basis, and the estimation step can be implemented on a simple platform (wireless sensor) with computational limitations, as showed by [4].

The validity of a retardation model has been demonstrated by having a single sensor estimating the distance to, and speed of, a passing propeller-driven aircraft, see [3].

There are also examples of sensor networks with multiple sensors that use Doppler shift for source localization. A way to do this is to model the measured frequency difference (in effect of the Doppler shift) among every sensor pair in the network. This rich set of measurements may then be informative enough to identify more complex motion models, for instance ballistic trajectories, see [8]. Another (suboptimal) way to configure the network is to have each sensor make a local estimate of the passage distance and speed, and then transmit the results to a fusion node that in turn aggregates the individual estimates to a more informative output in terms of global trajectory coordinates. This was demonstrated by [2] and later by [9]. If the state of the source is a priori known at some instant, Doppler based source tracking can be accomplished by calculating a frequency stretch factor between pairs of distributed microphones. This technique is proposed in [10], where the authors demonstrate aircraft tracking with a network of seven microphones. The aircraft state may then be assumed known at take-off or touch-down by means of ground control cameras.

There are many reasons for keeping the number of sensors in a network moderate, such as purchase, deployment, and maintenance costs. Balancing costs, in terms of sensor count, with performance is an essential part of the sensor system design that requires careful analysis. For instance, with as few as five Doppler sensors (with a non-degenerate 
geographical distribution) it is possible to uniquely and instantaneously determine the source position, see [11].

The scientific contribution of this work includes the analysis of a new circular motion model for acoustic Doppler measurements that accounts for acoustic propagation delays. It is not sensitive to retardation effects since the propagation delay is explicitly modeled. The model is more general or flexible than the frequently proposed linear motion models, but also more complex to identify. Most models in the literature referenced above are based on linear motion. We have also studied linear models to confirm the results of earlier works and to investigate how these can be extended to sensor network settings. Parts of these studies we published in [12], but since the linear motion is just a special case of the model we propose here, we have however chosen not to include these studies in this paper. The linear models are not appropriate at all for the non-linear motion used in the numerical experiments.

The estimator will here be developed as the solution to a least squares problem which is pursued by numerical minimization. However, the objective function is not generally convex. Particularly, when an acoustic source is observed for only a few seconds by a limited number of sensors operating at low signal-to-noise ratio, it is challenging to find a robust initialization that yields a successful minimization. We recently proposed the new model in [12]. The news in this work is 1) a robust solution to the initialization problem and 2) error analysis which is based on extensive Monte Carlo simulations.

The identification is formulated as a batch processing problem, which means that the source has more or less passed the sensors before the parameters are estimated. This is suitable for implementation on lightweight platforms (wireless sensor nodes) in applications where a delay of a few seconds is acceptable. This does not necessarily restrict the method to off-line uses. All on-line system solutions are in fact associated with various degrees of response delays attributable to processing time, transmission time and so forth. The point is that the proposed method could very well be used in on-line systems, but may not be the best choice in situations where the response delay is critical. An application could be a ground sensor network that from a limited set of alternatives decides which way passing vehicles take. Another application is a sensor that can determine the trajectory of passing aircraft $[3,9,10]$ or rockets [4]. Also passive sonar has been mentioned in the literature. Moreover, we presented a tracking application for multiple ground vehicles with a probability hypothesis density (PHD) filter using Doppler measurements in a acoustic sensor network, see [13]. For more references on Doppler-based tracking we refer to that work.

The paper is organized as follows. Next, in Section 2, the estimation problem is formalized and modeled, and the general assumptions made are described. Then a method to efficiently initialize the resulting optimization problem is detailed Section 3, followed by a tailored Gauss-Newton method to solve the estimation problem in Section 4. Section 5 then briefly discusses implications in the context of sensor networks, before the suggested method is validated, in Section 6, using both Monte Carlo simulations and experiments on real data from an all-terrain vehicle. Finally, conclusions are provided in Section 7. 


\section{Signal Model}

The Doppler model is based on the source-observer propagation delay, $\Delta(t)$, which leads to a comprehensive description of the relation between source motion and observed Doppler shift, both in the time and frequency domain. By our definition, the propagation delay is with respect to the observed signal or observer time frame, and it is assumed that it satisfies

$$
\Delta(t) \triangleq \operatorname{sol}_{\tau>0} F(\tau)=0, \text { where } F(\tau) \equiv \tau-\frac{1}{c} r(t-\tau),
$$

$c f$. [5]. Here, $c$ is the assumed known speed of sound and $r(t)$ is the source-observer distance. The solution (solubility) of this equation depends on source motion (how the motion is modeled). It is further assumed that a single moving target emits an acoustic signal (engine noise) with constant but unknown frequency $f$. The target approaches our sensor network from afar, passes through or at some distance from the network, and then departs. Our sensor network has $M \geq 2$ synchronized microphones with known locations $s_{i}, i=1,2, \ldots, M$. Sensor $i$ detects the signal, tracks the observed frequency, and produces the Doppler frequency measurement

$$
y_{i}(t)=f \cdot\left(1-\dot{\Delta}\left(t ; \theta, s_{i}\right)\right)+\varepsilon(t),
$$

where $\varepsilon(t)$ is a Gaussian process with constant spectral density up to half the sampling frequency, $\frac{1}{2 T}$, and no energy above. Dot notation, “ $\dot{\Delta}(t)$ ”, is used to denote time derivative. The motion gives rise to a Doppler shift that is modeled by $\Delta\left(t ; \theta, s_{i}\right)$, where $\theta$ comprises the unknown parameters of the target motion. The problem in focus is to identify the target motion by solving the least squares (LS) problem

$$
\hat{\theta}=\underset{\theta}{\arg \min } \min _{f} \sum_{i, j}\left(y_{i}(j T)-f \cdot\left(1-\dot{\Delta}\left(j T ; \theta, s_{i}\right)\right)\right)^{2} .
$$

As mentioned in the introduction, this is a batch formulation of the problem, which means that measurements from the whole passage is collected before attempting to identify the motion.

Consider for a moment the special case with strict radial motion, $r(t)=v t, v$ being the constant source speed. Then (1) is solved by $\Delta(t)=v t /(c+v)$, and $\dot{\Delta}(t)=v /(c+v)$. In this special case, $f \cdot\left(1-\dot{\Delta}\left(j T ; \theta, s_{i}\right)\right)$ gives the classical expression for Doppler shift.

Not all acoustic sources are easily casted to the ideal sinusoidal assumed here. It is, however, somewhat out of scope to detail how this preprocessing step should be performed in general in this paper. Instead, the experimental section demonstrates one possible way to approach this step for an all-terrain vehicle with a harmonic acoustic spectrum, see Figure 6.

\subsection{The Circular Motion Model}

The estimation of the source motion is based on the parameterized motion model $\Delta\left(t ; \theta, s_{i}\right)$, which is a mapping from the motion parameter $\theta$ to propagation delay, given the sensor location. The motion is assumed to be circular with unknown radius. Of course, this model does not allow the target to move around freely, but considered as a 
method that identifies the motion of sources that temporarily pass during a few seconds, it is believed to be general enough for many of the applications earlier mentioned. The circular target path is described by the function $p(t)$, which in turn is a function of the rotation angle $\alpha(t)$. The unknown parameters are; the signal frequency $f$, $\operatorname{target}$ speed $v$, initial heading $\alpha_{0}=\alpha(0)$, initial position $p_{0}=p(0)$, and inverse radius $\zeta$, see Figure 1 . By choosing the inverse radius $\zeta$, the parameterization is smooth around the straight line motion. A parameter transition from convex curvature, over straight line to concave curvature can be performed without discontinuities. This choice facilitates an efficient numerical search for optimal parameter values.

\section{[Figure 1 about here.]}

By assuming that the source and sensors are all restricted to the ground plane, there are no more than six unknown parameters; (nuisance) $f$ and the five sought motion parameters

$$
\theta=\left[\begin{array}{lllll}
v & \alpha_{0} & p_{0, x} & p_{0, y} & \zeta
\end{array}\right]^{T} .
$$

The plane restriction may be suitable for the application of ground sensors for ground vehicle monitoring, in which case moderate altitude variations do not contribute significantly to the estimation error. Airborne sources, on the other hand, would call for a three-dimensional model with eight parameters and minor model generalizations that should be trivial to the practitioner. However, in this work we focus on the plane model. Define for notational convenience the rotated unit vector and orthogonal operator as

$$
R(\alpha) \triangleq\left[\begin{array}{c}
\cos \alpha \\
\sin \alpha
\end{array}\right], \quad R_{\perp}(\alpha) \triangleq\left[\begin{array}{c}
-\sin \alpha \\
\cos \alpha
\end{array}\right] .
$$

By fundamental trigonometric arguments, the source location is then

$$
p(t)=p_{0}-\frac{1}{\zeta} R\left(\alpha_{0}\right)+\frac{1}{\zeta} R\left(\alpha_{0}+t \zeta v\right) .
$$

Note that an equivalent form, although less intuitive, that avoids potential numerical problems in the limit $\zeta \rightarrow 0$ (movement along straight line) is

$$
p(t)=p_{0}-t v\left[\begin{array}{l}
\cos \alpha_{0} \sin \frac{t \zeta v}{2} \operatorname{sinc} \frac{t \zeta v}{2}+\sin \alpha_{0} \operatorname{sinc} t \zeta v \\
\sin \alpha_{0} \sin \frac{t \zeta v}{2} \operatorname{sinc} \frac{t \zeta v}{2}-\cos \alpha_{0} \operatorname{sinc} t \zeta v
\end{array}\right],
$$

where $\operatorname{sinc} \alpha \triangleq \lim _{\alpha^{\prime} \rightarrow \alpha} \frac{\sin \alpha^{\prime}}{\alpha^{\prime}}$. The distance and relative speed between the source and the $i$ th sensor have the forms

$$
\begin{aligned}
& r_{i}(t)=\sqrt{\left(p(t)-s_{i}\right)^{T}\left(p(t)-s_{i}\right)}, \\
& \dot{r}_{i}(t)=v \cdot \frac{\left(p(t)-s_{i}\right)^{T} R_{\perp}\left(\alpha_{0}+t \zeta v\right)}{\sqrt{\left(p(t)-s_{i}\right)^{T}\left(p(t)-s_{i}\right)}} .
\end{aligned}
$$

The propagation delay $\Delta\left(t ; \theta, s_{i}\right)$ is implicitly given by (1). Even though the motion model and source location are given on closed form, all attempts to obtain a closed form expression for $\Delta\left(t ; \theta, s_{i}\right)$ have failed. Therefore we propose solving the propagation delay numerically using the Newton's method, see [14, pp. 58] for details. Note first that

$$
\Delta\left(t ; \theta, s_{i}\right) \approx \frac{1}{c} r_{i}(t)
$$


is an approximate solution that is obtained by ignoring the retardation, that is, the stop-and-go approximation referred to in the introduction. As long as the speed of the source is considerably lower than that of sound, this is a fairly good approximation. Even though the error is not always negligible, the approximation has proved to serve well to initialize the Newton iterations. The implementation of Newton's method to solve (1) is given by

$$
\begin{aligned}
\Delta^{(0)} & =\frac{1}{c} r(t), \\
\Delta^{(k+1)} & =\Delta^{(k)}-\frac{F\left(\Delta^{(k)}\right)}{F^{\prime}\left(\Delta^{(k)}\right)} \\
& =\Delta^{(k)}-\frac{c \Delta^{(k)} r\left(t-\Delta^{(k)}\right)-r^{2}\left(t-\Delta^{(k)}\right)}{c r\left(t-\Delta^{(k)}\right)+v\left(p\left(t-\Delta^{(k)}\right)-s_{i}\right)^{T} R_{\perp}\left(\alpha_{0}+\left(t-\Delta^{(k)}\right) \zeta v\right)} .
\end{aligned}
$$

The progress of the algorithm can be monitored by evaluating $F\left(\Delta^{(k)}\right)$, which should decrease in each iteration. In our experiments, convergence to machine precision is typically attained after as few as two iterations which indicates that the Newton iterations are well behaved for this problem. Once given $\Delta\left(t ; \theta, s_{i}\right)$, the chain rule gives in turn

$$
\dot{\Delta}\left(t ; \theta, s_{i}\right)=\frac{d}{d t} \frac{1}{c} r\left(t-\Delta\left(t ; \theta, s_{i}\right)\right)=\frac{\dot{r}_{i}\left(t-\Delta\left(t ; \theta, s_{i}\right)\right)}{c+\dot{r}_{i}\left(t-\Delta\left(t ; \theta, s_{i}\right)\right)} .
$$

The stop-and-go approximation $\Delta\left(t ; \theta, s_{i}\right) \approx \frac{1}{c} r_{i}(t)$ basically means that the Newton iterations are omitted. Another, rougher model simplification is of course to disregard the acoustic propagation delay altogether. Simulations will later show the impact of these approximations.

\section{Initial Estimate}

To our knowledge, (3) can for the current application only be solved by iterative numerical algorithms. It is, however, observed that (3) is generally not free from suboptimal local minima, a fact that complicates the numerical approach significantly and makes the initial estimate an important issue. The initial estimate $\theta^{(0)}$ proposed here is derived in three steps:

(i) extract basic signal features;

(ii) solve an initial LS problem; and

(iii) transform the initial parameter estimate $\hat{\phi}$ to $\theta^{(0)}$.

The idea with a different model for the initialization is to relax the coupling between the sensors and solve an estimation problem that is almost on the individual-sensor level. One could compare it to the extreme, where there would be as many models and parameter vectors as there are sensors, although we do not take it that far. Instead, the relaxed problem is formulated in a way that the source frequency $f$, speed $v$, and radius $1 / \zeta$ are common to all sensors, while the closest point of approach, as identified by the passage distance $d_{i}$ and passage time $\kappa_{i}$ (both constants), are individual, see Figure 1. The parametrization in $d_{i}$ and $\kappa_{i}$ is used, instead of other alternatives, as it allows for an efficient 
solution to the initalization problem, as outlined below. The obviously over-parameterized motion parameter vector is then

and the initial model defined by

$$
\phi=\left[\begin{array}{lllllll}
v & \zeta & d_{1} & \kappa_{1} & \ldots & d_{M} & \kappa_{M}
\end{array}\right]
$$

$$
y_{i}(t)=f \cdot\left(1-\dot{\Delta}\left(t ; \phi_{i}\right)\right)+\epsilon_{i}(t),
$$

where $\phi_{i}=\left[\begin{array}{lllll}f & v & \zeta & d_{i} & \kappa_{i}\end{array}\right]$ is used to simplify the notation. Basically the solution to the initial problem

$$
\hat{\phi}=\underset{\phi}{\arg \min } \min _{f} \sum_{i, t}\left(y_{i}(t)-f \cdot\left(1-\dot{\Delta}\left(t ; \phi_{i}\right)\right)\right)^{2}
$$

is pursued here. Compared to the original LS problem, this LS problem is much easier to initialize. Here it will be outlined how to initialize $\phi_{i}$ based on four signal features $\left(f_{a, i}, f_{b, i}, l_{1, i}, l_{2, i}\right)$ that are computed with a few simple operations on $y_{i}(t)$ for each sensor (step (i)). Typically, this feature extraction gives rise to a number of initial estimate candidates to be explored. If the measurements are particularly noisy, the robustness of the initial estimates can be improved by at least partially solving (14) (step (ii)). Finally in step (iii) of the initialization, (12) is adapted to (4) in LS-sense, and the LS-residuals at this step indicate which candidates to test in the final estimation round.

\subsection{Simplified Model for Initialization}

Assume that $\zeta \geq 0, d_{i} \neq 0$ (the trajectory does not pass through the sensor), and by definition $d_{i} \geq-\frac{1}{\zeta}$, see Figure 1 . These assumptions are made to avoid singularities and ambiguities in the coming derivation. The singular case when the source passes exactly through the sensor is here assumed to be of limited interest. To derive $\dot{\Delta}\left(t ; \phi_{i}\right)$, let the Cartesian source and fix, relative, sensor coordinates be formulated as

$$
\begin{aligned}
p(t) & =\frac{1}{\zeta} R(v \zeta t), \\
w_{i} & =\left(\frac{1}{\zeta}+d_{i}\right) R\left(v \zeta \kappa_{i}\right),
\end{aligned}
$$

respectively. This is distinct from the earlier parameterization in that the source-sensor angle depends individually on $\kappa_{i}$ instead of collectively on $\alpha_{0}$. Furthermore, at this stage the knowledge of the sensor position $s_{i}$ is disregarded. Instead the relative sensor coordinates $w_{i}$ depend solely on the value of the parameter vector. The source-sensor distance follows as

$$
\begin{aligned}
r_{i}(t) & =\sqrt{\left(p(t)-s_{i}\right)^{T}\left(p(t)-s_{i}\right)} \\
& =\sqrt{\frac{2 v^{2}\left(t-\kappa_{i}\right)^{2}\left(1+d_{i} \zeta\right) \operatorname{sinc}^{2}\left(v \zeta\left(t-\kappa_{i}\right)\right)}{1+\cos \left(v \zeta\left(t-\kappa_{i}\right)\right)}+d_{i}^{2}}
\end{aligned}
$$

and the derivative as

$$
\dot{r}_{i}(t)=\frac{\left(1+d_{i} \zeta\right)\left(t-\kappa_{i}\right) v^{2} \operatorname{sinc}\left(v \zeta\left(t-\kappa_{i}\right)\right)}{r_{i}(t)} .
$$

The stop-and-go approximation $\Delta(t ; \phi) \approx \frac{1}{c} r_{i}(t)$ is certainly good for an initial estimate and the combination with (18) and (11) immediately gives the sought $\dot{\Delta}\left(t ; \phi_{i}\right)$. 


\subsection{Step (i), Extract Basic Signal Features}

For the special case $d_{i}>0, \zeta>0$ (the sensor is placed outside the circular motion circumference), distinct extrema of $\dot{r}_{i}(t)$ are attained when

$$
\cos \left(v \zeta\left(t-\kappa_{i}\right)\right)=\frac{1}{1+d_{i} \zeta}
$$

is satisfied, at which instants the motion is directly towards/away from the sensor. This can either be derived geometrically, or by setting the derivative of (18) to zero, and solving the so obtained equation analytically. This yields the extreme values

$$
\begin{aligned}
& \max _{t} f \cdot\left(1-\dot{\Delta}\left(t ; \phi_{i}\right)\right)=\frac{f}{1-\frac{|v|}{c}}, \\
& \min _{t} f \cdot\left(1-\dot{\Delta}\left(t ; \phi_{i}\right)\right)=\frac{f}{1+\frac{|v|}{c}} .
\end{aligned}
$$

The frequency extrema are apparent in the example M3 of Figure 3(a). On the other hand, for $d_{i}<0$ and $\zeta>0$ (sensor placed inside the circular motion circumference), the received frequency extremes are attained when

$$
\cos \left(v \zeta\left(t-\kappa_{i}\right)\right)=1+d_{i} \zeta
$$

is satisfied, which can be deduced by analyzing the geometry in place or again by taking the derivative of (18) and setting it to zero and look for solutions. For these solutions, the extreme values are

$$
\begin{aligned}
& \max _{t} f \cdot\left(1-\dot{\Delta}\left(t ; \phi_{i}\right)\right)=\frac{f}{1-\frac{|v|}{c}\left(1+d_{i} \zeta\right)}, \\
& \min _{t} f \cdot\left(1-\dot{\Delta}\left(t ; \phi_{i}\right)\right)=\frac{f}{1+\frac{|v|}{c}\left(1+d_{i} \zeta\right)},
\end{aligned}
$$

which conforms with the intuition that the Doppler shift decreases as the sensor approaches the center of the target motion, see the example M1 and M2 of Figure 3(a). Provided that the Doppler shift is observed sufficiently long time before and after the passage instant $t=\kappa_{i}$, the signal features $f_{a, i}=\max _{t} y_{i}(t) \approx \max _{t} f \cdot\left(1-\dot{\Delta}\left(t ; \phi_{i}\right)\right)$ and $f_{b, i}=\min y_{i}(t) \approx \min _{t} f \cdot\left(1-\dot{\Delta}\left(t ; \phi_{i}\right)\right)$ approximate these extremes rather well and can be obtained with very simple computational means. Then, for $d_{i}>0$,

$$
\begin{aligned}
\frac{|v|}{c} & =\frac{f_{a, i}-f_{b, i}}{f_{a, i}+f_{b, i}}, \\
f & =\left(1-\left(\frac{|v|}{c}\right)^{2}\right) \frac{f_{a, i}+f_{b, i}}{2},
\end{aligned}
$$

and for $d_{i}<0$

$$
\begin{aligned}
\left(1+d_{i} \zeta\right) \frac{|v|}{c} & =\frac{f_{a, i}-f_{b, i}}{f_{a, i}+f_{b, i}}, \\
f & =\left(1-\left(\left(1+d_{i} \zeta\right) \frac{|w|}{c}\right)^{2}\right) \frac{f_{a, i}+f_{b, i}}{2} .
\end{aligned}
$$


In both cases,

$$
f \approx \frac{1}{2}\left(f_{a, i}+f_{b, i}\right)
$$

Apparently, $f_{a, i}$ and $f_{b, i}$ are both simple and informative signal features, and more of these can be extracted at the passage instant $t=\kappa_{i}$ when $y_{i}\left(\kappa_{i}\right) \approx f \cdot\left(1-\dot{\Delta}\left(\kappa_{i} ; \phi_{i}\right)\right) \approx f$. Note that the second derivative $\ddot{r}_{i}\left(\kappa_{i}\right)=-\frac{|v|^{2}}{\left|d_{i}\right|}\left(1+d_{i} \zeta\right)$ identifies the slope of the frequency curve at the passage,

$$
\left.\frac{d}{d t} f \cdot\left(1-\dot{\Delta}\left(t ; \phi_{i}\right)\right)\right|_{t=\frac{\left|d_{i}\right|}{c}}=\frac{f}{c} \ddot{r}_{i}\left(\kappa_{i}\right)
$$

The entity $\frac{\left|d_{i}\right|}{c}$ here accounts for the acoustic propagation delay from closest point of approach to $w_{i}$. A simple line fit,

$$
\min _{l_{1, i}, l_{2, i}} \sum_{t}\left(y_{i}(t)-l_{1, i}-l_{2, i} t\right)^{2}
$$

to a few frequency measurements (at least coarsely) associated with the passage is found to be a rather robust approach to find the slope as $l_{2, i}$, which thus identifies (26). Typically four or five consecutive measurements are suitable for this line fit, selected symmetrically around the zero crossing of $y_{i}(t)-f$. Put together,

$$
\begin{aligned}
\frac{\left|d_{i}\right|}{c} & =\frac{-f\left(\frac{|v|}{c}\right)^{2}}{l_{2, i} \pm f\left(\frac{|v|}{c}\right)^{2} \zeta} \\
\kappa_{i} & =\frac{f-l_{1, i}}{l_{2, i}}-\frac{\left|d_{i}\right|}{c} .
\end{aligned}
$$

Basically all identities above also hold for the linear motion case, $\zeta=0$, although then there will be no distinct frequency extrema; maximum and minimum are then formally replaced by supremum and infimum, but can still be approximated by the maximum and minimum values of $y_{i}(t)$ in practice. A more serious implication of $\zeta=0$ is that the sign of $d_{i}$ becomes unobservable. To deal with this, a set of initial solutions with permuted signs may be considered. When later exposing these candidate solutions to the knowledge of the sensor locations, these ambiguities may be resolved. The sign of $v$, which apparently is not observed at this stage, is deduced more or less automatically when later transforming $\phi$ to $\theta$.

Above is outlined how the features $f_{a, i}, f_{b, i}, l_{1, i}$, and $l_{2, i}$ relate to $d_{i}, \kappa_{i}, f, \zeta$, and $v$. In the multisensor perspective, each sensor contributes to the equation system, where the unknowns are $f$ and the elements of $\phi$. Logic has to be implemented to organize the solving efficiently, and as mentioned, more than a few candidate solutions may need to be considered. However, with two or more sensors present, with at least one circumscribed, $d_{i}<0$ and $\zeta>0$, there are at least as many equations as there are unknowns, and a unique solution is thus enabled. If the Doppler $\operatorname{shift}\left(f_{a, i}-f_{b, i}\right)$ varies among the sensors, this is a certain indication that this is the state of matter. If no such variation is present, then there are two hypothesis to exploit. Either $d_{i}<0$ or $d_{i}>0$ for each sensor. In neither case $\zeta$ can be deduced from the proposed features, and one would have to resort to generate initial estimates based on guessing the value of $\zeta$ (for instance, $1 / \zeta \in\{100,200, \ldots, 6400\}$.) 


\subsection{Step (ii), Solve Initial LS problem}

By at least approximately solving (14), the initial estimate is refined in a way that improves the optimization success rate for high noise levels. A few iterations of a general optimization program is appropriate.

\subsection{Step (iii), Transform $\hat{\phi}$ to $\theta^{(0)}$}

$\hat{\phi}$ is apparently computed without using the knowledge of the sensor coordinates. $\hat{\phi}$ is rather a hypothesis of where the sensors are relative an equally hypothetic target motion and this hypothesis is based only on available measurements. $\theta$, on the other hand, primarily specifies the motion, and does so in absolute coordinates independently of the sensor positions. If the hypothesis is valid, there exists an affine transform that maps the hypothetical positions, $w_{i}$, to the real, $s_{i}$, and this may then also be applied to transform the hypothetical motion coordinates to absolute, which gives $\theta$. If no such transform exists, or if the best possible transform still gives a large residual error, then the hypothesis should be rejected, possibly in favor for some other hypothesis.

The hypothetical coordinates, $w_{i}$, are given by $\phi$ from (15) and the affine transformation $\{A, b\}$ is required to minimize the LS residual

$$
V(A, b)=\sum_{i=1}^{M}\left\|A w_{i}+b-s_{i}\right\|_{2}^{2},
$$

under the orthonormal constraint $A^{T} A=I$. Computing $A$ and $b$ is a problem with a well-know solution efficiently obtained with a singular value decomposition (SVD), see [15] for the details. Then

$$
\begin{aligned}
& p_{0}=A\left[\begin{array}{ll}
\frac{1}{\zeta} & 0
\end{array}\right]^{T}+b, \\
& \alpha_{0}=\operatorname{atan}\left(A_{1,2}, A_{1,1}\right),
\end{aligned}
$$

where atan $(\cdot, \cdot)$ is the quadrant compensated arctangent. If the SVD produced the mirrored solution, then $\operatorname{det} A=-1$ and the sign of $v$ and $\alpha_{0}$ need to be changed accordingly.

\section{Tailored Gauss-Newton}

Since the unknown frequency parameter $f$ enters the model equation linearly ( $f$ is separable), it can be replaced by its implicit least squares solution, which reduces the parameter dimension and facilitates a more efficient nonlinear optimization step. Formulate (2) on time discrete vector form as

$$
Y=f \cdot G(\theta)+E,
$$

where rows are sample instances, $G=G(\theta) \in \mathbb{R}^{n}$. Note that measurements from all sensors are stacked in $Y$, so the dimension $n$ sums all measurements from all sensors, that is, all terms in (3). Given any hypothesis on $\theta$, the (unweighted) least squares estimate of $f$ is

$$
\hat{f}=\left(G^{T} G\right)^{-1} G^{T} Y
$$


and inserted into (31) it gives

$$
Y=G\left(G^{T} G\right)^{-1} G^{T} Y+E .
$$

The least squares estimate of $\theta$ may now be pursued by minimizing

$$
\hat{\theta}=\underset{\theta}{\arg \min }\left\|\left(I-G\left(G^{T} G\right)^{-1} G^{T}\right) Y\right\|_{2}^{2} .
$$

General optimization software can be used for this, for instance the Nelder-Mead minimization algorithm implemented in the fminsearch function in MATLAB.

However, if efficiency is of any importance, attention should instead be given the more specialized Gauss-Newton algorithm, which has well-documented merits as a least squares problem solver, see [16, pp. 259]. For the separable case at hand, the forms of both the objective function and its Jacobian are, notably, different compared to the case with ordinary nonlinear least squares. In [17] it is proposed that (34) is equivalently reformulated as

$$
\hat{\theta}=\underset{\theta}{\arg \min }\|P(\theta) Y\|_{2}^{2},
$$

where $P=P(\theta)$ is orthogonal and spans exactly the nullspace of $G$. $P$ is computed by a $\mathrm{QR}$ factorization at each Gauss-Newton step. In [17] it is also proposed how to compute the Jacobian $J=\nabla(P Y) \in \mathbb{R}^{n \times p}$ required by the Gauss-Newton algorithm. This variation of the algorithm is termed variable projection (Varpro). In the special case at hand (with only one separable parameter), the Jacobian is well approximated by

$$
\nabla(P Y) \approx-P \nabla G G^{\dagger} Y
$$

where $G^{\dagger}$ is a symmetric generalized inverse of $G$. This computationally efficient approximation was proposed in [18]. A step in the algorithm updates the estimate as

$$
\hat{\theta}^{(k+1)}=\hat{\theta}^{(k)}-\left(K^{T} K\right)^{-1} K^{T} P Y,
$$

where $K=-P \nabla G G^{\dagger} Y$. Computing $\nabla G \in \mathbb{R}^{n \times 5}$ is (in the application at hand) the most expensive part of the algorithm, regardless if it is done analytically or by finite differences. The many computations needed at each step of the GaussNewton algorithm however pay off, see [12].

\subsection{Notes on Computational Complexity}

The amount of computations needed depends on the anticipated noise level and also on requirements on optimization success rate. Since the objective function is not convex in general, it is not possible to guarantee that the (optimal) solution is found. Of course, with negligible noise, even the basic features extracted in Step (i) in Section 3.2, Section 3, (converted in Step (iii) in Section 3.4), would probably give sufficient accuracy and to a very low computational cost. Also at moderate noise levels, Step (ii) in Section 3.3 can be skipped, although the final Gauss-Newton will make a significant improvement. Still, the computations take a fraction of a second to complete (running MATLAB on an office laptop). At higher noise levels, it becomes increasingly hazardous to dismiss initial hypothesis at an early stage, 
and even if all proposed hypotheses are tested through the final estimation step, there may still be a few percent of the realizations that result in failure. In this work we have prioritized robustness, so all hypotheses are pursued to the full before selecting the one with least residual. This takes roughly five seconds. In a practical implementation one would probably estimate the current noise level to be able to moderate the complexity and simultaneously deliver the required success rate.

\section{Network Distributed Estimation}

Although the estimation step proposed here is inherently centralized, there are natural ways to distribute the computations to the local sensor nodes in a way that saves bandwidth, provided the nodes have the processing ability required. If frequency detection and tracking modules are distributed, for instance, only frequency estimates at rather low rate need to be propagated. An eight bit frequency update sent twice a second (say) requires $16 \mathrm{bps}$ bandwidth per target and sensor, plus minor detection overhead.

Suboptimal approaches of distribution may be interesting to study, although we will not pursue them here. For instance, the bandwidth requirement could be even further reduced by having each node running a single sensor algorithm similar to the initial estimate previously described, and only send a few parameter estimates.

\section{Numerical Experiments}

\subsection{Monte Carlo Simulation}

Two scenarios are designed for testing the proposed model and LS estimator via Monte Carlo (MC) simulations, and later via real world experiments; one with motion radius $1 / \zeta=85 \mathrm{~m}$, and one with $1 / \zeta=2 \mathrm{~km}$. The same target speed, $v=14 \mathrm{~m} / \mathrm{s}$, and microphone configuration are used in both scenarios, see Figure 2. The received frequency is measured twice a second during $20 \mathrm{~s}$. The MC noise levels are chosen with respect to $f=100 \mathrm{~Hz}$. If, for instance, the equivalent performance at $f=200 \mathrm{~Hz}$ is sought, the used noise levels need to be multiplied by two. Figure 3 illustrates the ideal, normalized frequency curves, and also an example of noise perturbation with the $100 \mathrm{~Hz}$ equivalent level $\sigma=0.5 \mathrm{~Hz}$.

\section{[Figure 2 about here.]}

[Figure 3 about here.]

For each noise level, 4000 realizations are used to test the proposed algorithm; first the initial estimate then followed by the tailored Gauss-Newton iterations. The iterations stop when the relative objective function improvement from one iteration to the next falls below $10^{-5}$. To indicate whether the optimization is successful, a clairvoyant estimate initialized by the true parameter is produced for reference. If the clairvoyant residual is smaller than that of the estimate, the realization is counted as a failure. Otherwise the estimate contributes to the root mean square error 
(RMSE) statistics that are presented as results. Since we prioritize robustness here, as many as eight initial hypotheses are always evaluated full before selecting one that minimizes the residual.

\subsection{Results, Monte Carlo Simulation}

The resulting RMSE for the motion parameters as a function of the noise level $\sigma$ is given in Figure 4, and the fraction of optimization (or rather initialization) failures is given in Table 1. No significant estimation biases are detected. In Figure 4 also the Cramér-Rao Lower Bound (CRLB, see [19]) are plotted for comparison. Note that CRLB is throughout this work presented in terms of standard deviations (std), not variance. Apparently, the algorithm is numerically efficient up to $\sigma \approx 0.5$ (at $f=100 \mathrm{~Hz}$ ). For $\sigma \gg 0.5$ the parameter RMSE departs significantly from the CRLB, particularly for the speed $(\hat{v})$ and initial position $\left(\hat{p}_{0}\right)$ estimates in the $1 / \zeta=85 \mathrm{~m}$ scenario. From Table 1 it is seen that the initial estimate degrades rather gracefully with increased noise level. Apparently, the motion curvature $(1 / \zeta=85 \mathrm{~m})$ makes the initialization more sensitive to noise.

\section{[Figure 4 about here.]}

[Table 1 about here.]

Although more subtle, the computational efficiency limit (as far as the two scenarios studied are concerned) is probably also in the interval $\sigma=0.5$ through $\sigma=1$. Higher noise levels obviously mean more frequent initialization failures, but also more difficult to early dismiss initial candidates, by for instance (29), simply because any model mismatch is dominated by noise. More hypothesis testing with an increasing share of numerically pathological cases, leads to drastic increase in computational requirements.

\subsubsection{Model Simplifications}

When using the stop-and-go approximation $\Delta(t) \approx \frac{1}{c} r(t)$ (disregarding the retardation effect), a small relative degradation is noticed in MC simulations at the lower noise levels, which should have little practical implication in the studied case. It however can have a more attenuated negative effect in degenerated sensor-source constellations, where the solution is sensitive. On the other hand, disregarding the acoustic propagation delay altogether will result in a noticeable bias. In Figure 5, $4000 \mathrm{MC}$ simulations on the original model is compared to ditto on a model that ignores the delay, and some half meter bias is then apparent. These model simplifications have rather limited positive effect on the solution time. The main benefit is most likely that a simplified set of equations is more manageable with respect to coding and memory use on restricted low-level platforms.

\section{[Figure 5 about here.]}

[Figure 6 about here.] 


\subsection{ATV Localization Data Set}

Real data from an all-terrain vehicle (ATV) is recorded with the same two setups as in the simulated scenarios described previously. The measurements are described in detail in [20]. A single track for each scenario is used to prove the concept. The acoustic sampling rate is $2 \mathrm{kHz}$ and frequency estimates are taken twice a second in discrete time windows. The ATV engine sound is characterized by a fundamental frequency at approximately $50 \mathrm{~Hz}$ with distinct harmonics. The sound pressure is estimated to be $110 \mathrm{~dB}$ SPL and the background noise level to be $58 \mathrm{~dB}$ SPL in the utilized frequency range $30-1000 \mathrm{~Hz}$. Sensors are perfectly synchronized, and sensor positions are known to the decimeter. ATV ground truth reference tracks are measured with an accurate receiver for the global navigation satellite system (GNSS). The horizontal error including any receiver displacement is appreciated to be at the level of $1 \mathrm{~m}$.

\subsubsection{Frequency Estimation}

In this work, frequency estimation and motion estimation are separate processing steps, where we have so far entirely focused on the latter. How the frequency estimation is approached in practice depends on the source characteristics. Here a brief description is given how we process the ATV sound.

Sources like ATVs, motorcycles, and propeller driven aircraft often have a distinct harmonic structure, a fact that is utilized in this numerical experiment. The spectrogram for the ATV sound is given in Figure 6 as an illustration. The ATV apparently have spectral components in a rather wide frequency range $50-350 \mathrm{~Hz}$, which may give the estimation robustness, while each individual harmonic component is rather narrow band, which facilitates efficient processing based on finding peaks in a periodogram.

The frequency estimation is conducted in $25 \%$ overlapping Hamming time windows that are centered around the time instants of the frequency samples, $j T$. Let $F_{j}\left(\varphi_{q}\right)$ be the discrete-time Fourier transform of window $j$, with indexed frequency $\varphi_{q}$. The sensor index $i$ is here omitted. The frequency measurement is now given by

$$
y(j T)=\underset{f_{1}<\varphi_{q}<f_{2}}{\arg \max } \sum_{h=1}^{n_{h}} \sum_{l} w_{l}\left|F_{j}\left(\varphi_{q h+l}\right)\right|
$$

where $w$ is a smoothing frequency window,

$$
\left[\begin{array}{lll}
w_{-1} & w_{0} & w_{1}
\end{array}\right]=\left[\begin{array}{lll}
0.5 & 1 & 0.5
\end{array}\right] .
$$

$f_{1}$ and $f_{2}$ restrict the search to the region where the fundamental frequency has been detected; $n_{h}$ is the number of harmonics including the fundamental frequency. This frequency measurement thus relies on multiple harmonic components, $\varphi_{q}, \varphi_{2 q}, \ldots, \varphi_{n_{h}}$, which makes it robust to partial losses (or drop in the signal-to-noise ratio) of the frequency spectrum, even to a temporary black-out of the fundamental component. The discrete-time Fourier transform has very efficient computational implementations, although it fails to capture the (Doppler) frequency variation within each time window. It is thus numerically suboptimal. 


\subsection{Results, ATV Data Set}

Figure 7 gives a visual comparison between estimated trajectory and the ground truth (GNSS) for the $1 / \zeta=85 \mathrm{~m}$ and $2 \mathrm{~km}$ motion scenarios, respectively. Figure 8 compares the measured (and normalized) frequency $y_{i}(t)$ with the estimate $h\left(t ; \hat{\theta}, s_{i}\right)$. A numeric comparison of parameter values is given in Table 2, where also the CRLB based on the model residuals (RMSE $=0.43$ and $0.24 \mathrm{~Hz}$, respectively) is noted. CRLB is worse here compared to the simulated scenarios, since the signal durations are significantly shorter $(\ll 20 \mathrm{~s})$. The diagram in Figure 9 shows how the absolute localization error evolves over time. As the ATV passes the central part of the network, the errors attain minimum; approximately $4-5 \mathrm{~m}$.

The parameter errors are possibly acceptable, but yet significant. It is noted that the data set is not ideal, since there are significant fluctuations in the engine frequency, which of course violates the model assumption.

[Figure 7 about here.]

[Figure 8 about here.]

[Figure 9 about here.]

[Table 2 about here.]

\section{Conclusion}

A circular motion model for localization of acoustic sources based on the Doppler shift observed in distributed microphones has been proposed. The model is accurate in the sense that it fully accounts for the retardation effect. A state-of-the-art nonlinear least squares estimator based on the computationally efficient Gauss-Newton variable projection technique has been applied to the estimation problem. An initial estimate based on simple and robust measurement features has been proposed as a start parameter for the Gauss-Newton, which is sensitive to local optima. The model and estimation scheme have been evaluated by Monte Carlo simulations and demonstrated on real data from an all-terrain vehicle (ATV).

Although there are no guarantee that the estimation algorithm is successful in a particular realization of Gaussian noise, it is concluded that the estimation problem is feasible for practical noise levels. In the studied examples, the initialization fails in less than $2 \%$ of the cases when the measurement error is as large as 1 std dev $\mathrm{Hz}$ for a source that give a total Doppler shift of $\pm 4 \mathrm{~Hz}$. With the proposed initial estimate, the Gauss-Newton typically converges in 3-4 iterations and gives, together with the proposed initial estimate, a computationally efficient algorithm for moderate noise levels. The estimator is also close to the Cramér-Rao lower bound for noise levels that should be realistic in many practical situations.

With parts of the signal processing distributed to local sensor nodes, the bandwidth requirement for one sensor could be reduced to 16 bits per second and target. The techniques involved are fairly mature for practical implementations in a sensor network. 


\section{Acknowledgment}

This work is part of the project Cooperative Localization, COOP-LOC, at Linköping University, funded by the Swedish Foundation for Strategic Research (SSF) grant number RIT10-0051, and also part of research projects at the Swedish Defence Research Agency, FOI, that are funded by the Swedish Armed Forces.

\section{Bibliography}

[1] R. J. Webster, An exact trajectory solution from Doppler shift measurements, IEEE Transactions on Aerospace and Electronic Systems AES-18 (2) (1982) 249-252. doi:10.1109/TAES.1982.309235.

[2] Y. T. Chan, J. J. Towers, Sequential localization of a radiating source by Doppler-shifted frequency measurements, IEEE Transactions on Aerospace and Electronic Systems 28 (4) (1992) 1084-1090. doi:10.1109/7.165370.

[3] B. G. Ferguson, A ground-based narrow-band passive acoustic technique for estimating the altitude and speed of a propeller-driven aircraft, Journal of Acoustical Society of America 92 (3) (1991) 1403-1407.

[4] J. I. Statman, E. R. Rodemich, Parameter estimation based on Doppler frequency shifts, IEEE Transactions on Aerospace and Electronic Systems AES-23 (1) (1987) 31-39. doi:10.1109/TAES.1987.313333.

[5] U. Orguner, F. Gustafsson, Target tracking with particle filters under signal propagation delays, IEEE Transactions on Signal Processing 59 (6) (2011) 2485-2495.

[6] M. A. Richards, Fundamentals of radar Signal Processing, McGraw-Hill, 2005.

[7] Y. T. Chan, A 1-D search solution for localization from frequency measurements, IEEE Journal of Oceanic Engineering 19 (3) (1994) 431437. doi:10.1109/48.312919.

[8] E. Weinstein, N. Levanon, Passive array tracking of a continuous wave transmitting projectile, IEEE Transactions on Aerospace and Electronic Systems AES-16 (5) (1980) 721-726. doi:10.1109/TAES.1980.308936.

[9] D. C. Torney, Localization and observability of aircraft via Doppler shifts, IEEE Transactions on Aerospace and Electronic Systems 43 (3) (2007) 1163-1168. doi:10.1109/TAES.2007.4383606.

[10] S. R. Martín, M. Genescà, J. Romeu, T. Pàmies, Aircraft tracking by means of the acoustical doppler effect, Aerospace Science and Technology 28 (1) (2013) $305-314$.

[11] I. Shames, A. Bishop, M. Smith, A. Brian, Analysis of target velocity and position estimation via Doppler-shift measurements, in: Australian Control Conference, Melbourne/Australia, 2011, pp. 507-512.

[12] D. Lindgren, M. B. Guldogan, F. Gustafsson, H. Habberstad, G. Hendeby, Acoustic source localization in a network of Doppler shift sensors, in: 16th International Conference on Information Fusion, Istanbul, Turkey, 2013.

[13] M. B. Guldogan, D. Lindgren, F. Gustafsson, H. Habberstad, U. Orguner, Multi-target tracking with PHD filter using doppler-only measurements, Digital Signal Processing 27 (0) (2014) 1 - 11.

[14] K. E. Atkinson, An introduction to numerical analysis, 2nd Edition, Wiley, 1989.

[15] K. S. Arun, T. S. Huang, S. D. Blostein, Least-squares fitting of two 3-d point sets, IEEE Transactions on Pattern Analysis and Machine Intelligence 9 (5) (1987) 698-700.

[16] J. Nocedal, S. J. Wright, Numerical Optimization, Springer, New York, 1999.

[17] G. Golub, V. Pereyra, The differentiation of pseudo-inverses and nonlinear least squares problems whose variables separate, SIAM Journal on Numerical Analysis 10 (2) (1973) 413-432.

[18] L. Kaufman, A variable projection method for solving separable nonlinear least squares problems, BIT Numerical Mathematics 15 (1975) 49-57.

[19] S. M. Kay, Fundamentals of Statistical Signal Processing: Estimation Theory, Prentice-Hall, 1993. 
[20] V. Deleskog, H. Habberstad, G. Hendeby, D. Lindgren, Acoustic and visual sensor network measurements in Lilla Gra 2012, Tech. Rep. MEMO4833, The Swedish Defence Research Agency, FOI, Linköping, Sweden (2014)

URL http: //www. foi.se 


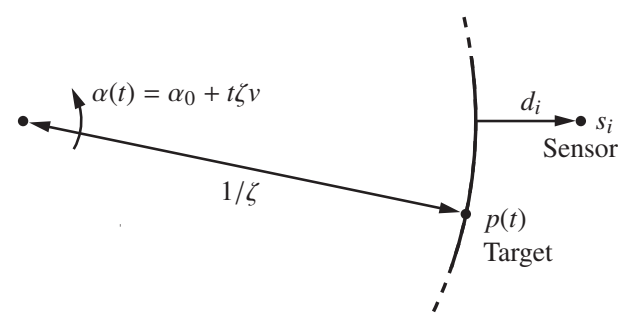

Figure 1: The target motion is assumed to be circular with unknown radius $1 / \zeta$. The speed $v$ is also unknown but assumed to be constant. The sensors are at known coordinates $s_{i}$ with unknown distances $d_{i}$ to the closest points of approach. $d_{i}$ are parameters in the simplified model for initialization. With $d_{i}<0$, sensor $i$ is inside the circle. Note that by definition $d_{i} \geq-\zeta^{-1}$, as the sensor position is assumed to be positioned on a ray out from the center of the circle. 


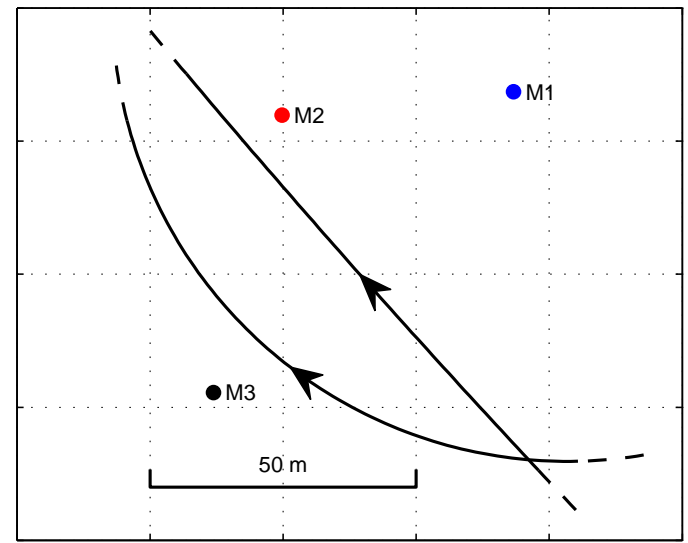

Figure 2: Two motion scenarios with a common sensor constellation consisting of three synchronized and omnidirectional microphones (M1-M3). In both scenarios the acoustic target follows circular paths, however paths with distinct radii; $1 / \zeta=85 \mathrm{~m}$ and $2 \mathrm{~km}$, respectively. (The curvature of the $2 \mathrm{~km}$ trajectory appears almost straight in this scale.) The target speed is $v=14 \mathrm{~m} / \mathrm{s}$. 


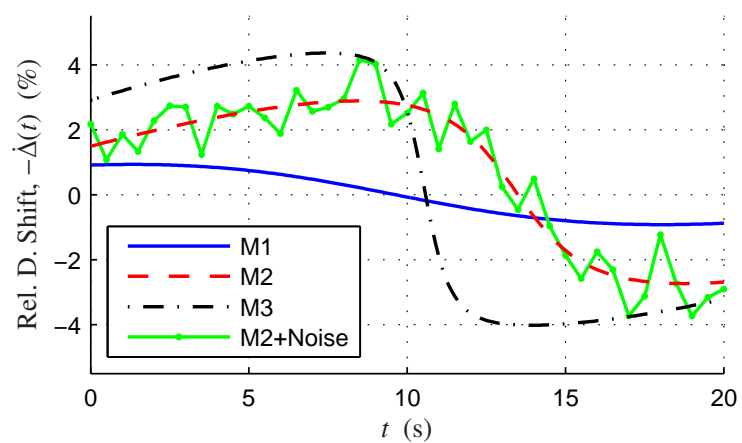

(a) Radius $1 / \zeta=85 \mathrm{~m}$

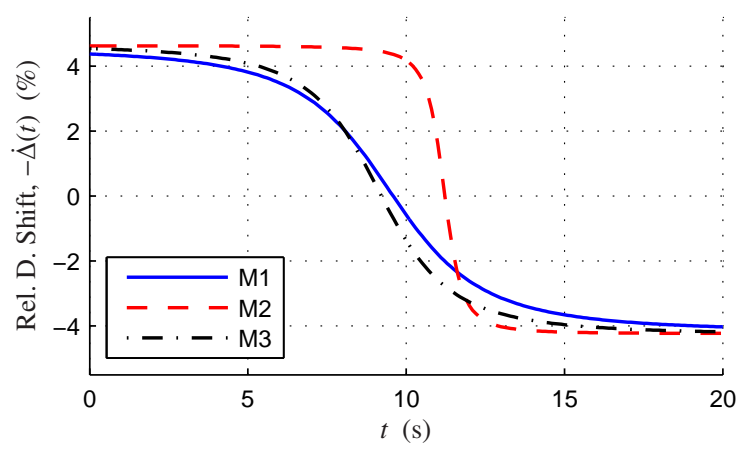

(b) Radius $1 / \zeta=2 \mathrm{~km}$

Figure 3: Theoretical Doppler shift at the three microphones (M1-M3) for the two motion radii in Figure 2. The Doppler shift is relative to the observed signal frequency. The $M 2+$ Noise plot illustrates frequency measurements at $\mathrm{M} 2$ of a $100 \mathrm{~Hz}$ source perturbed by noise with $\sigma=0.5 \mathrm{~Hz}$, two samples per second. 

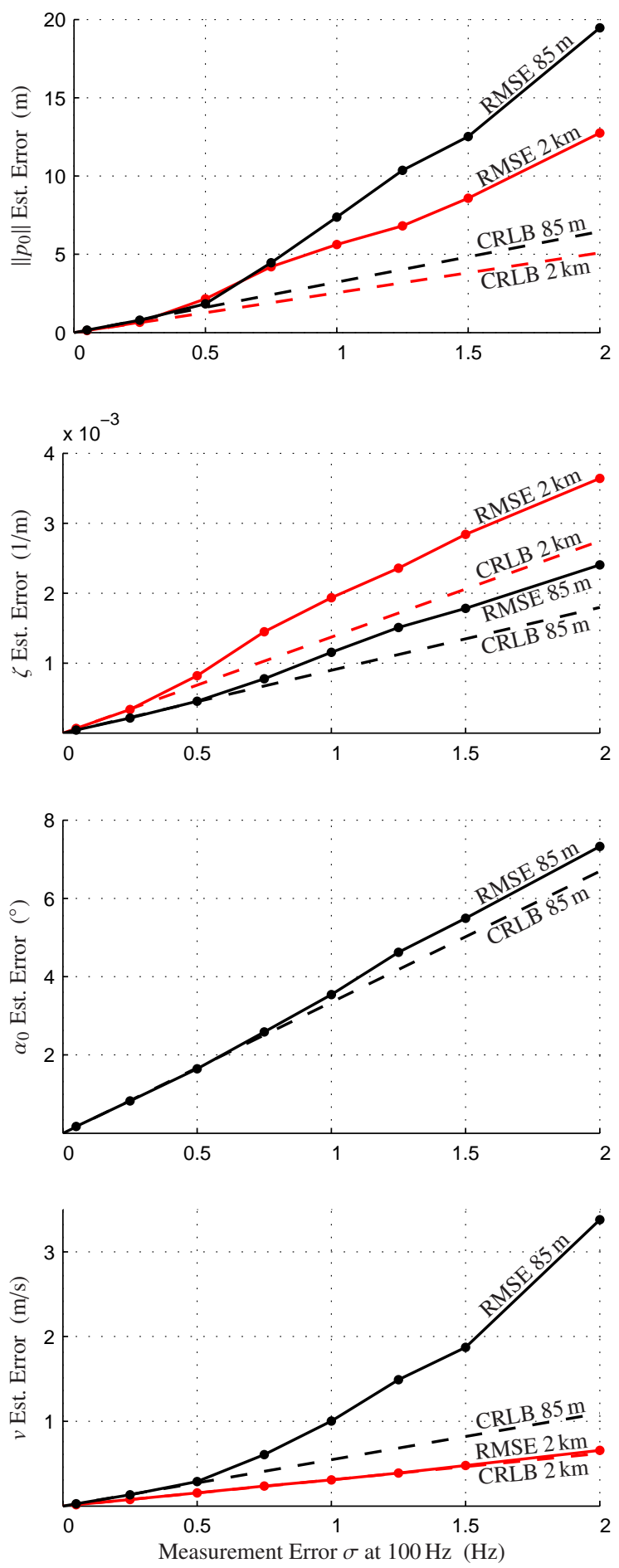

Figure 4: Monte Carlo simulation RMSE and CRLB versus the measurement error $\sigma$ given a sinusoidal target signal at $100 \mathrm{~Hz}$. Results for both the $1 / \zeta=85 \mathrm{~m}$ and the $2 \mathrm{~km}$ scenario. For $\alpha_{0}$ the results are indistinguishable between $85 \mathrm{~m}$ and $2 \mathrm{~km}$, so only $85 \mathrm{~m}$ is shown. Each point in the diagram is based on 4000 Monte Carlo runs. 


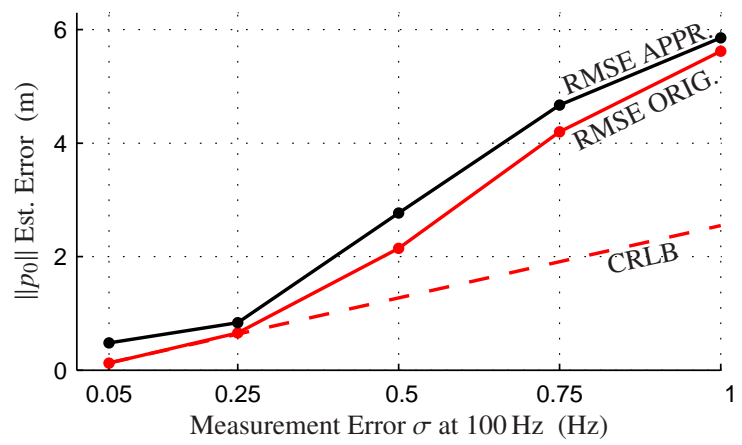

Figure 5: Penalty for disregarding the propagation delay. The RMSE from $5 \times 4000$ Monte Carlo runs on the $1 / \zeta=2 \mathrm{~km}$ scenario (RMSE APPR) shows that the accuracy of the $p_{0}$ coordinate degrades approximately $0.5 \mathrm{~m}$ compared to the original model (RMSE ORIG). 


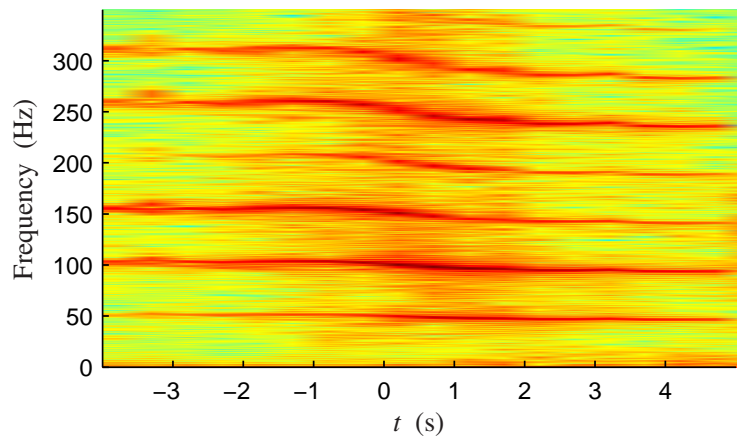

Figure 6: Acoustic spectrogram from microphone M3 for the ATV in the $1 / \zeta=85 \mathrm{~m}$ scenario. The engine revolutions give distinct harmonics that are used in the frequency estimation. 


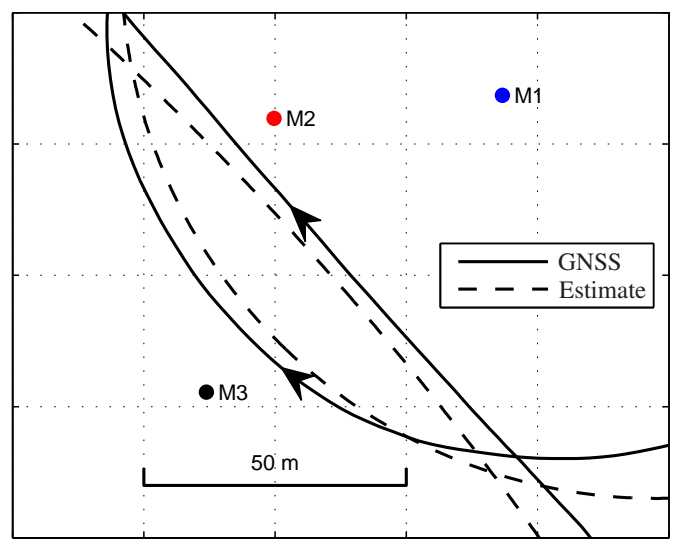

Figure 7: Estimated trajectory compared to ground truth (GNSS). The localization error is 4-5 $\mathrm{m}$ as the target passes through the sensor network. 


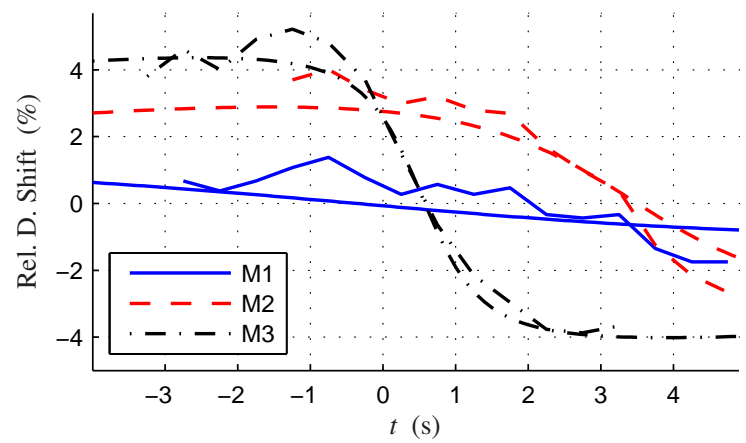

(a) $1 / \zeta=85 \mathrm{~m}, V(\hat{\theta})=0.43($ at $100 \mathrm{~Hz})$

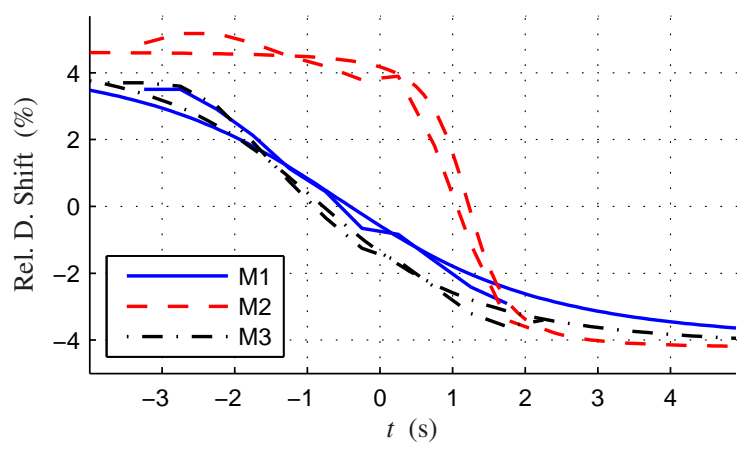

(b) $1 / \zeta=2 \mathrm{~km}, V(\hat{\theta})=0.24($ at $100 \mathrm{~Hz})$

Figure 8: Frequency measurements $y(t)$ (rugged curves) compared to the best fit model $h\left(t ; \hat{\theta}, s_{i}\right)$ (smooth curves) for the two ATV data sets. 


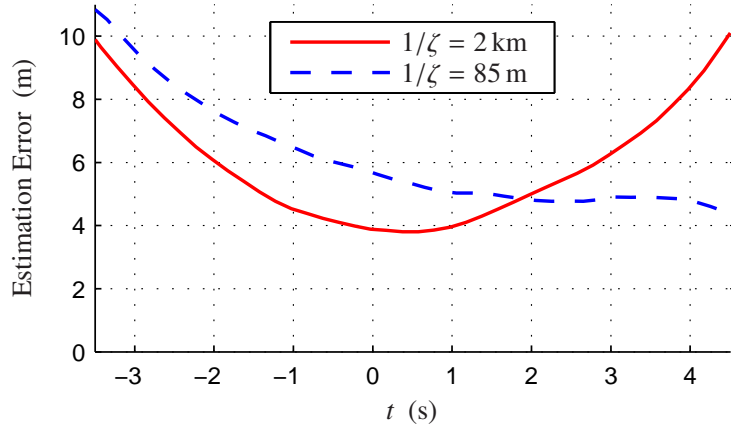

Figure 9: Absolute ATV localization error over time based on the reference GNSS measurements for the two scenarios. 
Table 1: Optimization (or initialization) failures in percent of 4000 Monte Carlo runs for the two example scenarios, and for different levels of the measurement error $\sigma$. A failure means that at better LS solution was found by initializing the optimization with the true parameter vector, or that rank deficiency were detected in the Gauss-Newton iterations.

\begin{tabular}{ccccccccc}
$\sigma$ at $100 \mathrm{~Hz}$ & 0.05 & 0.1 & 0.25 & 0.5 & 0.75 & 1 & 1.5 & 2 \\
\hline $1 / \zeta=85 \mathrm{~m}$ & 0 & 0 & 0.4 & 1.4 & 1.7 & 1.9 & 3.0 & 4.4 \\
$1 / \zeta=2 \mathrm{~km}$ & 0 & 0 & 0 & 0 & 0.1 & 0.4 & 1.0 & 1.7
\end{tabular}


Table 2: Parameter errors and the CRLB for the ATV data set. The CRLB is based on the model residual, $V(\hat{\theta})$, and on a ground truth parameter vector that is computed from accurate GNSS measurements.

\begin{tabular}{ccccc} 
& $\begin{array}{c}v \\
(\mathrm{~m} / \mathrm{s})\end{array}$ & $\begin{array}{c}\alpha_{0} \\
\left({ }^{\circ}\right)\end{array}$ & $\begin{array}{c}\zeta \\
(1 / \mathrm{km})\end{array}$ & $\begin{array}{c}\left\|p_{0}\right\| \\
(\mathrm{m})\end{array}$ \\
\hline $1 / \zeta=2 \mathrm{~km},|\hat{\theta}-\theta|$ & 1.0 & 1.4 & 2.1 & 3.1 \\
$1 / \zeta=2 \mathrm{~km}, \mathrm{CRLB}$ & 0.18 & 0.89 & 0.70 & 0.70 \\
$1 / \zeta=85 \mathrm{~m},|\hat{\theta}-\theta|$ & 0.99 & 3.8 & 1.9 & 4.0 \\
$1 / \zeta=85 \mathrm{~m}, \mathrm{CRLB}$ & 0.32 & 1.9 & 1.0 & 3.9
\end{tabular}

